\title{
Uniaxial Tension Testing Lab: Fewer Instructions for Better Results?
}

\section{Dr. Hadas Ritz, Cornell University}

Hadas Ritz is a senior lecturer in the Sibley School of Mechanical and Aerospace Engineering at Cornell University. She teaches required and elective courses covering a wide range of topics in the undergraduate Mechanical Engineering curriculum. Her main teaching interests include solid mechanics and finite element analysis. Ritz was recognized with a 2013 Cornell College of Engineering Excellence in Teaching Award. She received her PhD in Mechanical Engineering from Cornell in 2008.

Prof. Meredith N. Silberstein, Cornell University

Prof. Nelly Andarawis-Puri, Cornell University 


\section{Uniaxial tension testing lab: fewer instructions for better results?}

\section{Introduction}

At Cornell University, the engineering distribution course Statics and Mechanics of Solids is required for the mechanical engineering, civil engineering, biological engineering, and biomedical engineering departments. Each year roughly 350 students take this course, typically during their sophomore year. Two sections are offered each fall semester and one section each spring, meaning three lecturers are responsible for presenting the course each year. In 2015 the authors undertook a redesign effort whose goals have been to increase student learning outcomes regardless of the lecturer, and to motivate students by connecting course content to future engineering work. Part of that redesign effort, described further below, is the introduction of several benchtop labs where the students work in pairs using simple equipment to study course topics. The current study is focused on one of these labs: uniaxial tension testing. The fall 2017 students each performed one of two versions of this lab. One version followed a fully-guided approach where the lab document clearly laid out each step of the experiment. The other version more closely followed a discovery approach and focused more on student design of experiments, where the lab document specified the end goal (measurement of Young's modulus for a provided specimen) and described the equipment available, but did not provide instructions on data collection or analysis. To determine any outcome differences between these versions, students completed anonymous surveys following the labs and one question on a midterm exam specifically focused on the lab topic. In this paper, we briefly describe the overall course redesign, provide details about the two versions of the uniaxial tension testing lab, then present and discuss the data collected and results of our analysis.

In the past, this statics and mechanics of materials course involved three 50-minute lectures each week, one 50-minute discussion section each week led by a teaching assistant (TA), and three 2hour labs spread across the semester. During the fall semester when two lecture sections are offered, students share all other aspects of the course regardless of lecture section: discussion sections, lab sections, homework, exams. The main active learning component of the class has typically been iClicker use during lectures. The course redesign included four main thrusts: example problem videos, application videos with homework problems, in-lecture activities, and benchtop labs. Each of these innovations addresses common student complaints about the course. The example problem videos are full, detailed solutions of more complicated problems than those that can be done during the 50-minute lecture sessions. They are in response to the frequent complaint of disconnect between the difficulty or complexity of lecture examples versus homework problems. To demonstrate the relevance of course material to a variety of engineering fields, several homework problems were written based on interviews with alumni who now work in different industries, such as lithographic chip making and forklift manufacturing. For each of these problems the students watch a short video clip which provides brief background on the company and presents an engineering issue related to the course material. The homework assignment then fleshes out the problem from the video, using specific values, and sometimes including a limited design component. In-lecture activities also connect course material to topics beyond those covered in the textbook, and serve the dual role of incorporating more active 
learning into the course. These are full- or half-lecture long activities where the instructor presents a broad question and then leads the class step-by-step, using clicker questions, to the solution. The students work in small groups with their neighbors to solve each part, and course staff circulate through the room guiding progress. The final aspect of the redesign (and the relevant portion for this paper) transformed the lab portion of the course from a separate section in a dedicated lab room to smaller, more frequent "benchtop" labs that the students now perform during discussion sections. This is the most significant change to the structure of the course, and it has several benefits: the course no longer requires instructional support staff to maintain complicated laboratory equipment and train TAs on running each experiment; TA resources can be used to make smaller discussion section class sizes; all students in the course complete each lab during a single week instead of, due to overall class enrollment, having each experiment take several weeks; students get more individual interaction with the experiments instead of running black box equipment. These benchtop labs let the students study physical examples of current course content more frequently throughout the semester.

The two versions of the uniaxial tension lab compared during the fall 2017 semester are at different places on the spectrum from fully-guided learning to discovery learning $[1,2]$. One version arguably falls squarely in the fully-guided category. However, as described more fully below, the second version does not entirely fit the description of discovery learning. Kirschner and Sweller have argued [3] that discovery approaches are not effective for novices and result in less transferable understanding, although several authors [4-6] dispute their inclusion of problem-based learning and similar approaches in the broad category of discovery-based learning. The second version of the lab considered here does not require students to generate the concept of material stiffness or come up with a definition based on stress and strain. It can better be described as an experimental design exercise. Cooper [7] has found that for some groups of students, participating in experimental design improves understanding and retention. Etkina, et. al. [8] have found some evidence that student design of experiments may increase knowledge transfer. Rutar and Mason [9] showed that student perception of laboratory effectiveness and performance on related assignments can both be improved by designing experiments. In this study we use a targeted exam problem to assess whether the lab version impacted knowledge retention.

\section{Current study}

In the fall 2017 semester, approximately 230 students were enrolled in the course. Students were divided into 12 discussion sections, each with between 15-22 students. During the fifth week of the semester students did the uniaxial tension test lab during discussion sections. The concepts of stress and axial strain were introduced in the two lectures immediately preceding the lab. The idea of axial stiffness being related to a material property, Young's modulus, was briefly introduced in the lecture just before labs began. During the sixth week of the semester students took the first midterm exam. One problem, worth 10 points (out of 100 for the entire exam), directly tested understanding of the measurements and data analysis from the lab.

The students' goal for the uniaxial tension testing lab is to measure Young's modulus of a thin rubber specimen. The material and specimen dimensions were chosen to have easily measurable deformation under small load (the lab uses hanging weights of 100 to 600 grams) while still 
demonstrating a linear response on the stress-strain curve. The equipment for each pair of students includes: a rubber specimen ( $20 \mathrm{~mm}$ wide, $25 \mathrm{~cm}$ long, $1.5 \mathrm{~mm}$ thick) with two reference lines marked, a set of calibrated weights $(2 \times 100 \mathrm{~g}, 2 \times 200 \mathrm{~g})$, a rod stand, a transparent ruler to measure deflection of the reference lines, a hook to hang the weights from the specimen end, and a binder clip to hang specimen and ruler from the rod stand.

During the lab sessions, each pair of students was given a hard copy of the lab handout. For fall 2017 the first six sections to meet were given the open-ended version (OE) of the lab handout, which is included as Appendix A. The remaining six sections were given the step-by-step version ( $\mathrm{SbS}$ ) of the lab handout, which is included as Appendix B. The sections were divided in this manner (chronologically) to prevent students in the OE sections from seeing their classmates' SbS versions of the lab handouts. As much as possible, the goal was to have the students in OE sections design their own methodology, as described below. In all, based on section enrollment, approximately 110 students did the OE version and 120 students did the SbS version. The first page of both versions is identical: it lays out the goals of the lab activity, reminds students of the concepts and relevant equations, and includes an equipment list. The relevant equations are:

$$
\sigma=\frac{P}{A}
$$

where $\sigma$ is normal stress in an axially loaded member, $P$ is axial load, and $A$ is cross-sectional area;

$$
\epsilon=\frac{\delta}{L}
$$

where $\epsilon$ is normal strain, $\delta$ is elongation, and $L$ is initial specimen length; and

$$
E=\frac{\sigma}{\epsilon}
$$

is used to calculate Young's modulus, $E$. The next part of the lab handout describes the lab activity itself, and this is where the two versions diverge.

The SbS version instructs students to measure and record the cross-section dimensions, then provides a table and instructions to record the position of each reference line under no load and then under the action of six different weights. It explicitly tells them to calculate the gauge length by subtracting the position of one mark from the other. Finally, there is one more table for calculating and recording the strain and stress at each load and a set of labeled axes for students to graph their stress-strain data. It ends with discussion questions.

The OE version tells students they must find a value of Young's modulus for the provided specimen. It describes how to use the binder clip to hang the ruler and specimen and suggests using the reference lines to define a gauge section. It includes the same labeled axes as the $\mathrm{SbS}$ version, and tells students to brainstorm with their partner about what data they will need to collect and what experimental procedure to undertake. Blank space is provided to make tables for data recording and analysis.

To evaluate any differences between the lab versions, students were asked to complete a brief anonymous survey after their lab sections. The survey asked them to identify which section they attended and rate the length of the lab for the time provided. It then had three statements that students were asked to rate on a Likert scale of Strongly agree, Agree, Neither agree nor 
disagree, Disagree, Strongly disagree, or Not applicable. The statements were: I found the uniaxial tension lab to be very valuable; I found this lab to be intellectually challenging; I enjoyed this lab. The survey concluded with an open response question for any other comments or feedback about the lab. Our hypothesis was that student completing the OE version may find the lab to be more challenging and therefore more interesting.

In addition to the anonymous student feedback, the first exam included a problem specifically related to the lab. That problem is shown in Figure 1. We have found that most students understand that Young's modulus describes material stiffness, which is broadly related to the slope of a force-displacement response. The most common mistake students make when first learning about Young's modulus is directly using the slope of a force-displacement plot instead of finding the slope of the stress-strain plot. We designed this exam problem specifically to catch that mistake. The provided figure shows applied force and specimen length. To find Young's modulus, students had to first calculate specimen elongation at one or more load levels, then find strain and stress using the provided cross-section area and the original length, which must also be read off the graph. Our hypothesis was that students who had to design their own data collection procedure (OE version) would better understand all the steps necessary to go from force and length to Young's modulus, whereas students who completed the SbS version would be more prone to just remembering Young's modulus as the slope of a plot. Each student's score on that problem was recorded, along with the discussion section in which he or she was enrolled. The exam scores were not matched to specific attendance during the uniaxial tension lab, but almost all students attended their assigned section in general, and there were few absences for this lab. One of the authors graded this problem for all students to ensure uniform application of the grading rubric.

1. You have discovered a new/unknown ductile metal during a walk along Cornell's beautiful trails. You decide to bring it back to lab and you trim the sample so that it has a square cross sectional area with side length of $2 \mathrm{~mm}$. You test the sample in uniaxial tension, resulting in the graph of applied load versus specimen length, shown below. Calculate Young's modulus for this material. (10pts)

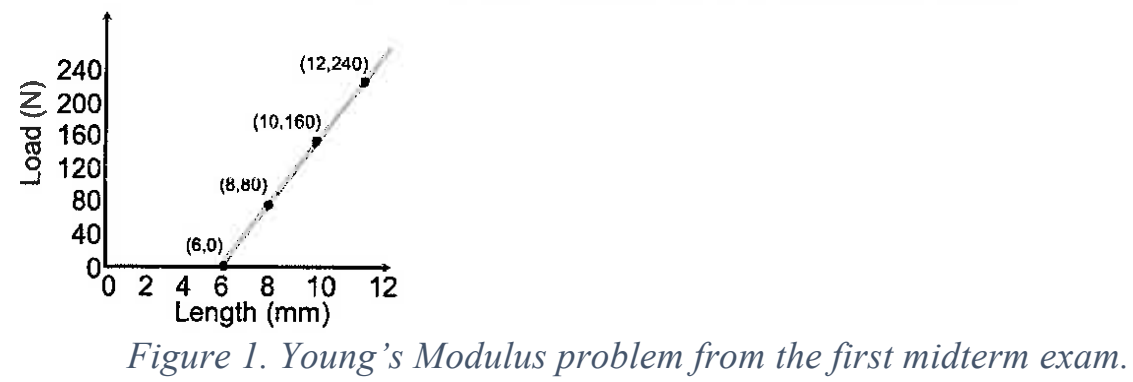

Results of both survey feedback and exam problem performance are presented in the following section.

\section{Results and discussion}

The anonymous survey responses showed no statistical significance in any differences between the OE and SbS versions of the lab. Figure 2 shows the survey results graphically. Tables 1-3 show the data as categorized for analysis via Fisher's exact test. For that test, we grouped "Strongly agree" and "Agree" into the single category "Agree." Similarly, "Strongly disagree" 
and "Disagree" are combined into the category "Disagree." That grouping, and the choice to use Fisher's exact test, is based on the small counts in the "Strongly agree" and "Strongly disagree" bins. All other responses are excluded from Fisher's exact test as performed here.

Of these negative results, perhaps the most surprising is that the students found no difference in the intellectual challenge of the lab depending on whether they simply followed step-by-step instructions or had to devise their own experimental procedure. This is counter to results seen by some researchers [9] where student perception of usefulness was positively influenced by a less guided approach. It is possible that the overall complexity of the lab is low enough that the challenge is perceived as minimal, even in the OE version. In survey comments, several students said that the lab was too easy, and some even suggested ways to make it more interesting (such as testing different materials, or different loading modes). Due to the nature of the lab logistics (taking place during 50-minute class periods, large deflection for small forces required since measurements are taken with rulers), it is not feasible to add that type of complexity. In the future, homework problems may be added that explore stress-strain curves for different materials.
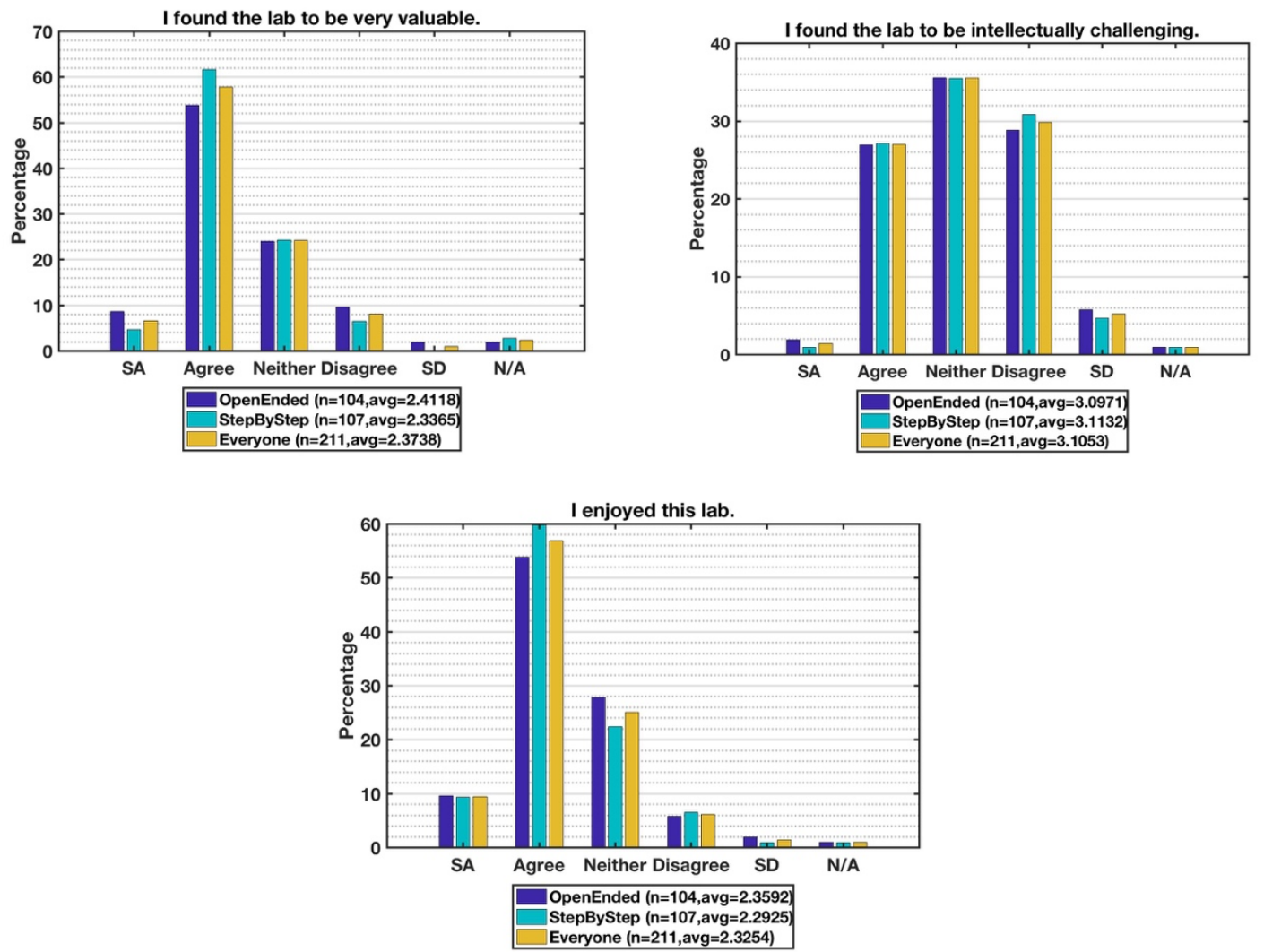

Figure 2: Likert scale survey results, separated by students completing the OE or SbS versions of the lab. The number of students contributing to each series is shown in the subfigure legends. The responses were assigned numerical values as follows: Strongly agree $=1$, Agree $=2$, Neither agree nor disagree $=3$, Disagree $=4$, and Strongly disagree $=5$. The averages reported in the legends are the means based on those values. 
Table 1: Responses to statement, "I found the uniaxial tension lab to be very valuable." as grouped for analysis.

\begin{tabular}{l|ccc} 
DATA ANALYZED & OE & SbS & TOTAL \\
\hline AGREE & 65 & 71 & 136 \\
DISAGREE & 12 & 7 & 19 \\
TOTAL & 77 & 78 & 155
\end{tabular}

Fisher's exact test: $p=0.2306$. No significant differences

Table 2: Responses to statement, "I found this lab to be intellectually challenging." as grouped for analysis.

\begin{tabular}{l|ccc} 
DATA ANALYZED & OE & SbS & TOTAL \\
\hline AGREE & 30 & 30 & 60 \\
DISAGREE & 36 & 38 & 74 \\
TOTAL & 66 & 68 & 134
\end{tabular}

Fisher's exact test: $p=1.0000$. No significant differences.

Table 3: Responses to statement, "I enjoyed this." as grouped for analysis.

\begin{tabular}{l|ccc} 
DATA ANALYZED & OE & SbS & TOTAL \\
\hline AGREE & 66 & 74 & 140 \\
DISAGREE & 8 & 8 & 16 \\
TOTAL & 74 & 82 & 156
\end{tabular}

Fisher's exact test: $p=1.0000$. No significant differences.

While student perception of the lab did not show significant differences between the two versions, scores on the associated exam problem did show statistically significant differences. The problem was worth a total of 10 points, 4 of which were awarded just for recognizing they needed to report some sort of a slope. The remaining 6 points were earned through calculating stress and strain, using appropriate units, and getting the correct final value. The most common reason for getting a score of 4 points was to take the slope of the plot as provided instead of converting to stress and strain. That is exactly the mistake we expected to be more common amongst students doing the SbS version, and indeed, while only $6 \%$ of OE students received 4 points on the problem, 14\% of SbS students received that score, as shown in Figure 3. 


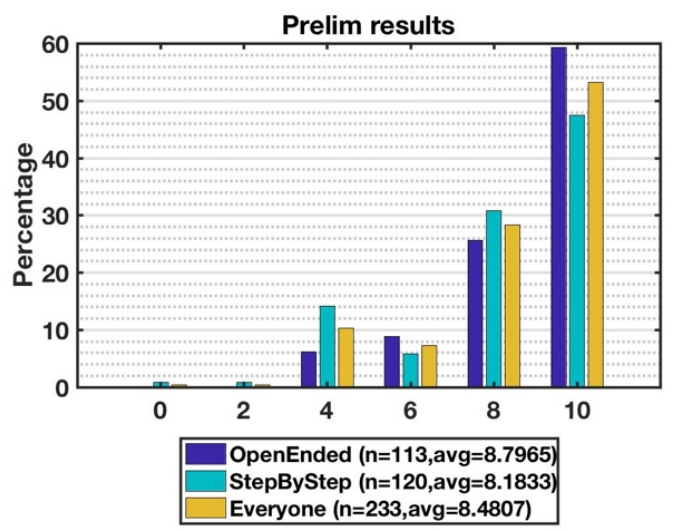

Figure 3: Student scores on prelim problem related to the uniaxial tension lab.

Overall students did very well on this simple exam problem, but even so, Fisher's exact test on the scores shows significance with $p=0.0223$. The data as analyzed for that test are given in Table 4. We separated the scores into below 5 points and above 5 points. Coincidentally, no student received a score of exactly 5 points on this exam problem. Since this test showed statistical significance, it warranted further analysis.

While exam problem score was found to have a statistically significant relation to lab version, the effect size is small, with Cohen's $d=0.322$ [10]. Since the students participating in each version of the lab were chosen solely based on discussion section timing, there is no reason to expect that "better" students were grouped into one version over another. However, we performed analysis of covariance using overall end-of-term course grade as the covariant variable. This is in lieu of student GPA, since those data were unavailable to us. The response variable was exam problem score and the independent variable was lab version. The results of that analysis are in Table 5, and show a significant interaction effect between the lab version and course score $(p=0.034)$. The nature of the interaction can be observed in Figure 4, which shows each student as well as linear least-squares fits to the two lab versions separately (which are included only to aid visualization). The effect of lab version was more evident for students with low overall course score, and this difference decreased as the course score increased. Nevertheless, the effect of being in the OE lab was an increase in scores for most of the students with less dependence on course score.

Table 4: Exam problem scores as categorized for Fisher's exact test.

\begin{tabular}{l|ccc} 
EXAM PROBLEM SCORE & OE & SbS & TOTAL \\
\hline LOWER THAN 5 & 7 & 19 & 26 \\
ABOVE 5 & 106 & 101 & 207 \\
TOTAL & 113 & 120 & 233
\end{tabular}

Fisher's exact test: $p=0.0223$. Cohen's $d=0.322$. 
Table 5: Analysis of covariance using exam problem score as the response variable, with lab version as independent variable and course score (CS) as covariant.

\begin{tabular}{l|ccccc} 
SOURCE & D.F. & SUM SQ & MEAN SQ & F & PROB $>$ F \\
\hline LAB & 1 & 33.4 & 33.4 & 8.98 & 0.003 \\
CS & 1 & 92.2 & 92.2 & 24.81 & $<0.001$ \\
LAB*CS & 1 & 16.8 & 16.8 & 4.53 & 0.0345 \\
ERROR & 217 & 806.4 & 3.7 & &
\end{tabular}

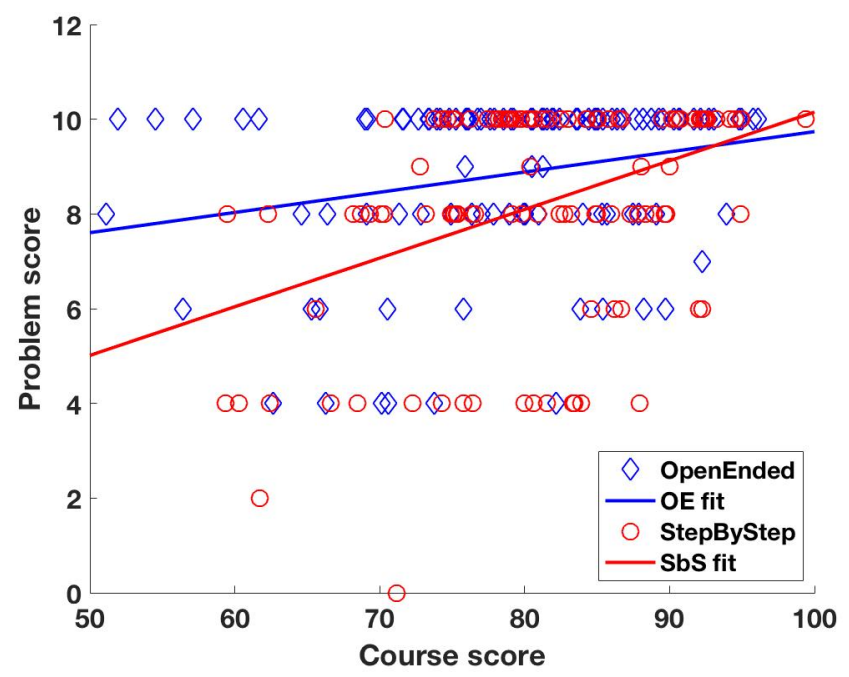

Figure 4: Exam problem score versus course score for each version of the lab.

\section{Conclusion}

In this study, we compared two versions of a simple uniaxial tension testing lab. We found that students' perception of the value and intellectual challenge of the lab, as well as their reported enjoyment, was not affected by whether step by step instructions were provided. However, students who completed the more open-ended version of the lab performed better one week later on an exam problem related to the lab concepts than did their peers who completed the step-bystep version $(p<0.05, d=0.32)$. Students who are generally weaker on this material, as judged by their eventual overall score in this course, tended to be helped more by the open-ended version of the lab than students who are generally stronger on this material. This outcome suggests that having to design their own experimental protocol may make students more likely to understand or remember all steps involved in particular data reduction tasks. When possible, instructors should avoid giving students unnecessarily detailed instructions. 
References

[1] J. S. Bruner, “The Art of Discovery," Harvard Educational Review 31 (1961): 21-32

[2] W. S. Anthony, "Learning to discover rules by discovery," Journal of Educational Psychology, 64(3), pp. 325-328, 1973.

[3] R. E. Clark, P. A. Kirschner, and J. Sweller, "Putting students on the path to learning: The case for fully guided instruction," American Educator, 36(1), 6-11, 2012.

[4] H. G. Schmidt, S. M. M. Loyens, T. van Gog, and F. Paas, "Problem based learning is compatible with human cognitive architecture: Commentary on Kirschner, Sweller, and Clark (2006)," Educational Psychologist, 42, 91-97, 2007.

[5] C. E. Hmelo-Silver, R. G. Duncan, and C. A. Chinn, "Scaffolding and achievement in problem-based and inquiry learning: A response to Kirschner, Sweller, and Clark (2006)," Educational Psychologist, 42, 99107, 2007.

[6] D. Kuhn, "Is direct instruction the answer to the right question?" Educational Psychologist, 42, 109-113, 2007.

[7] M. M. Cooper, "Cooperative chemistry laboratories," Journal of Chemical Education, 71(4), 307, 1994.

[8] E. Etkina, S. Murthy, and X. Zou, "Using introductory labs to engage students in experimental design," American journal of physics. Volume 74, Issue 11, pp. 986-986, 2006.

[9] T. Rutar, and G. Mason, "Design of experiments in introduction to thermodynamics course," Proceedings of the ASEE Annual Conference and Exposition, 2011.

[10] J. Cohen, "A Power Primer," Psychological Bulletin, vol. 112, (1), pp. 155-159, 1992. 


\section{Appendix A: Open ended (OE) version of the lab handout.}

Uniaxial Tension Test

Group Members:

\section{Goals:}

During this uniaxial tension test, you will:

- develop a better understanding of the concepts of stress and strain;

- learn how to measure elastic modulus;

- visualize strain localization and build intuition about Saint-Venant's principle.

\section{Introduction:}

Under uniaxial tension, the normal stress, $\sigma$, in the specimen can be found by:

$$
\sigma=\frac{P}{A}
$$

where $P$ is the applied force and $A$ is the cross section area. To measure the deformation of the material, we use normal strain, defined as:

$$
\epsilon=\frac{\delta}{L}
$$

where $\delta$ is the change in length and $L$ is the initial length. For linear elastic deformation, the stress is directly proportional to the strain and we define Young's (also called elastic) modulus, $E$, as:

$$
E=\frac{\sigma}{\epsilon}
$$

The rubber we will use is fairly linear at small strains but nonlinear at large deformation. In this experiment, we will use Eq. (3) to determine the Young's modulus for the material.

\section{Equipment:}

- One $1.5 \mathrm{~mm}$ thick rubber specimen, $20 \mathrm{~mm}$ wide, approximately $25 \mathrm{~cm}$ long.

- A set of calibrated weights $(2 \times 100 \mathrm{~g}$ and $2 \times 200 \mathrm{~g})$

- One rod stand with threaded screw clamp

- One flexible transparent ruler

- One small hook

- One binder clip

ENGRD 2020 Discussion Activity $\quad$ - 1 - 


\section{Activity:}

Using the provided equipment, you must find a value of Young's modulus for this rubber sample. The rubber specimen used in this experiment has two lines marked on it, as shown in the diagram below. These lines can be used to define a gauge section, which may help during data collection. The binder clip can be used to hang the rubber specimen from the rod stand.

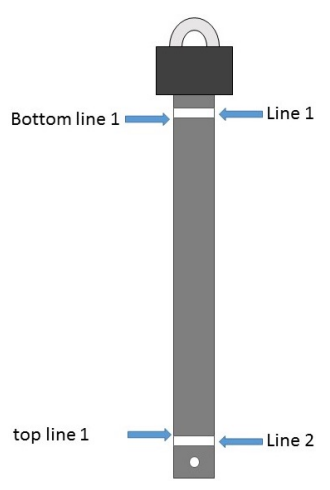

Figure 1: Overall schematic

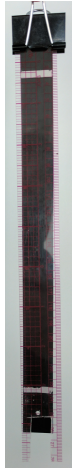

Figure 2: Ruler arrangement

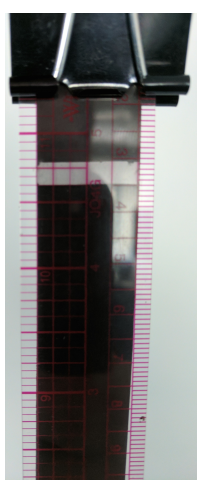

Figure 3: Line 1 position

Remember that Young's modulus is the slope of the linear portion of a material's stressstrain curve. You must use the figure below to plot stress-strain data for this material, and then find $E$ based on your plot.

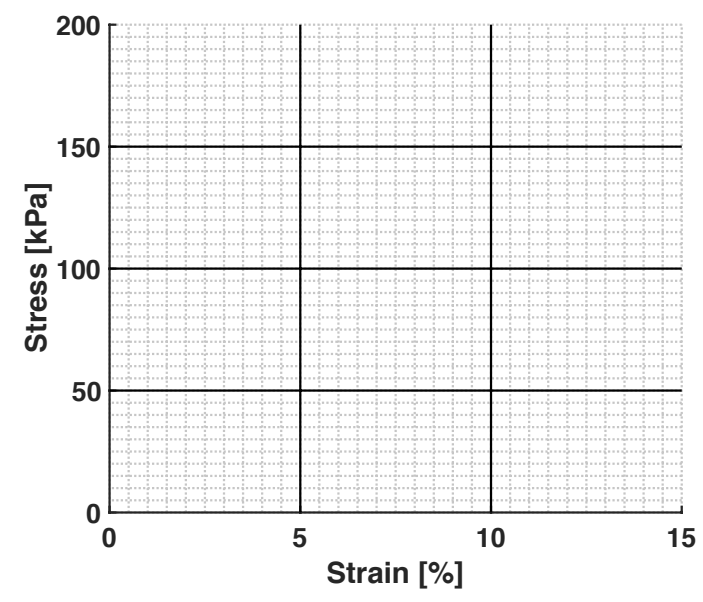

ENGRD 2020 Discussion Activity $\quad-\mathbf{2}-$ 
A1. Spend a few minutes brainstorming with your partner about what data you will need to collect. Use the space below to plan your experiments. For example, you may want to make an empty table which lists all the data you plan on collecting.

A2. Get approval from your TA of your experimental plan before proceeding.

ENGRD 2020 Discussion Activity $\quad-3-$ 
A3. Carry out your approved experimental plan, recording all data on the previous page, and ultimately plotting stress-strain data in the previously provided figure.

\section{Discussion:}

D1. Talk about the quality of your data. Do any of the data points look suspicious? If so, consider redoing those measurements.

D2. Based on the stress-strain curves, calculate the Young's modulus of this material. Note that the stress-strain curve may be nonlinear for strains above $5 \%$ due to the nonlinearity of the rubber material.

D3. Observe that deformation of the rubber specimen is nonuniform in the area near the any concentrated load (such as where the hook passes through the hole). The deformation far from the ends, however, is fairly uniform, allowing for more accurate strain measurements. Saint-Venant's principle says that if you move away from concentrated loads by a distance equal to the width of the specimen, the stress can be treated as uniform. Do your observations support that principle?

ENGRD 2020 Discussion Activity $\quad-4$ 


\section{Appendix B: Step by step ( $\mathrm{SbS}$ ) version of the lab handout}

Uniaxial Tension Test

Group Members:

\section{Goals:}

During this uniaxial tension test, you will:

- develop a better understanding of the concepts of stress and strain;

- learn how to measure elastic modulus;

- visualize strain localization and build intuition about Saint-Venant's principle.

\section{Introduction:}

Under uniaxial tension, the normal stress, $\sigma$, in the specimen can be found by:

$$
\sigma=\frac{P}{A}
$$

where $P$ is the applied force and $A$ is the cross section area. To measure the deformation of the material, we use normal strain, defined as:

$$
\epsilon=\frac{\delta}{L}
$$

where $\delta$ is the change in length and $L$ is the initial length. For linear elastic deformation, the stress is directly proportional to the strain and we define Young's (also called elastic) modulus, E, as:

$$
E=\frac{\sigma}{\epsilon}
$$

The rubber we will use is fairly linear at small strains but nonlinear at large deformation. In this experiment, we will use Eq. (3) to determine the Young's modulus for the material.

\section{Equipment:}

- One $1.5 \mathrm{~mm}$ thick rubber specimen, $20 \mathrm{~mm}$ wide, approximately $25 \mathrm{~cm}$ long.

- A set of calibrated weights $(2 \times 100 \mathrm{~g}$ and $2 \times 200 \mathrm{~g})$

- One rod stand with threaded screw clamp

- One flexible transparent ruler

- One small hook

- One binder clip

ENGRD 2020 Discussion Activity $\quad$ - 1 - 


\section{Activity:}

The rubber specimen used in this experiment has two lines marked on it, as shown in the diagram below. These lines define the gauge section and will be used in the data collection portion (Table 1). Be very careful to use the same mark for each measurement. For example, Mark 1 could be the bottom of Line 1, and Mark 2 could be the top of Line 2 .

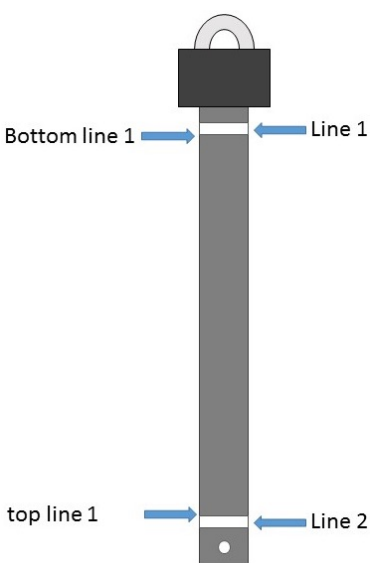

Figure 1: Overall schematic

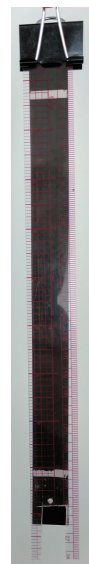

Figure 2: Ruler arrangement

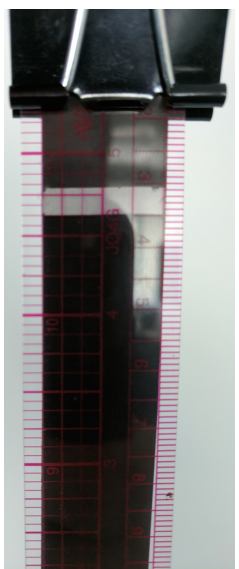

Figure 3: Line 1 position

You will measure the change in length of the gauge section to determine the strain. The calibrated weights will be used to apply the loads. The detailed steps are as follows:

A1. Measure and record the width of your specimen. Multiply by the thickness $(1.5 \mathrm{~mm})$ to get the cross-section area. Record both here.

Width:

mm Cross-section area:

$\mathrm{mm}^{2}$

A2. Clamp the ruler in front of the rubber specimen using the binder clip, as in Fig. 2 . The hole in the rubber should be in the slot in the bottom of the ruler. Position Line 1 on the specimen such that Mark 1 (whichever mark you've decided to use) is approximately aligned with the $3.5 \mathrm{~cm}$ mark, as in Fig. 3. Record the position of Mark 1 and Mark 2 in Table 1 . You will be precisely measuring (to the nearest $0.5 \mathrm{~mm}$ ) the position of each mark.

A3. Apply load by adding a $100 \mathrm{~g}$ mass to the hook at the bottom of the specimen. Both partners should look at the location of the marks and agree on the measurements. Record your findings in Table 1.

A4. Repeat for $100 \mathrm{~g}$ to $600 \mathrm{~g}$ to finish Table 1.

A5. By subtracting the measurements, calculate and record the gauge length in Table 2.

ENGRD 2020 Discussion Activity $\quad$ - $2-$ 
A6. Calculate the stresses and strains and record them in Table 2. Use $\mathrm{kPa}$ for the units of stress and percent for the units of strain.

A7. Plot stress versus strain in the provided grid.

\section{Data Collection:}

Table 1: Mark positions throughout loading.

\begin{tabular}{|c|c|c|}
\hline Mass $[\mathrm{g}]$ & Mark 1 $[\mathrm{mm}]$ & Mark 2 [mm] \\
\hline 0 & & \\
\hline 100 & & \\
\hline 200 & & \\
\hline 300 & & \\
\hline 400 & & \\
\hline 500 & & \\
\hline 600 & & \\
\hline
\end{tabular}

Table 2: Strain and stress calculated from data in Table 1. Note that the gauge section length for $0 \mathrm{~g}$ applied load is $L$, the original length, for strain calculation.

\begin{tabular}{|c|c|c|c|}
\hline Mass [g] & $\begin{array}{c}\text { Gauge section } \\
\text { length }[\mathrm{mm}]\end{array}$ & Strain [\%] & Stress [kPa] \\
\hline 0 & & & \\
\hline 100 & & & \\
\hline 200 & & & \\
\hline 300 & & & \\
\hline 400 & & & \\
\hline 500 & & & \\
\hline 600 & & & \\
\hline
\end{tabular}

ENGRD 2020 Discussion Activity $\quad-3-$ 


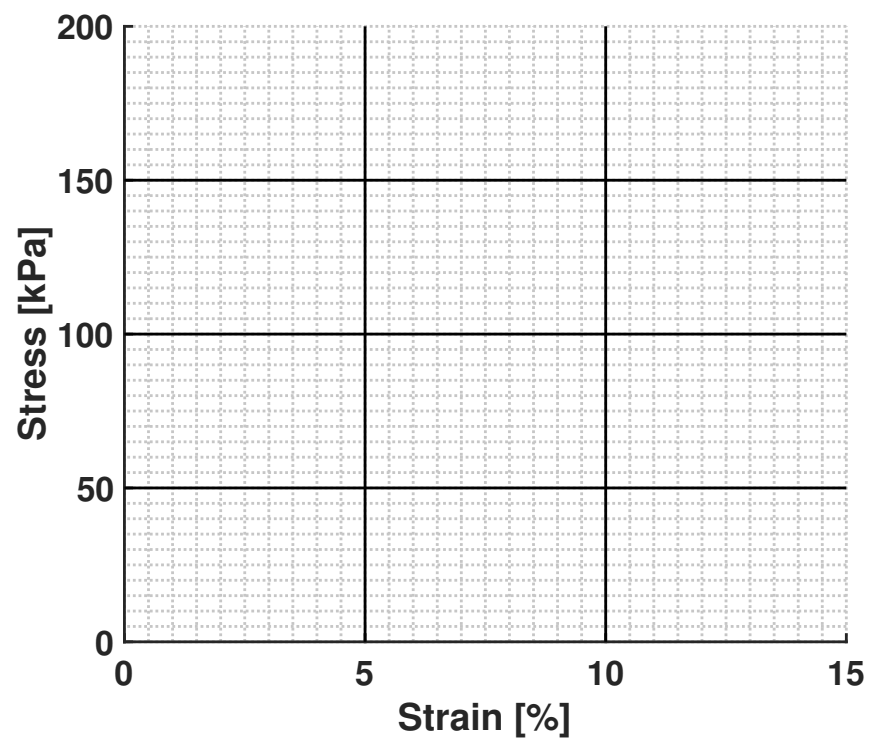

\section{Discussion:}

D1. Talk about the quality of your data. Do any of the data points look suspicious? If so, consider redoing those measurements.

D2. Based on the stress-strain curves, calculate the Young's modulus of this material. Note that the stress-strain curve may be nonlinear for strains above $5 \%$ due to the nonlinearity of the rubber material.

D3. Observe that the deformation is nonuniform in the area near the bottom end due to the concentrated load. The deformation far from the ends, however, is fairly uniform, allowing for more accurate strain measurements. Saint-Venant's principle says that if you move away from concentrated loads by a distance equal to the width of the specimen, the stress can be treated as uniform. Do your observations support that principle?

ENGRD 2020 Discussion Activity $\quad-4$ 